\title{
Weak Allee effect in a predator-prey system involving distributed delays
}

\author{
PAULO C.C. TABARES ${ }^{1 *}$, JOCIREI D. FERREIRA ${ }^{2 * *}$ \\ and V. SREE HARI RAO ${ }^{3 * *, * * *}$ \\ ${ }^{1}$ Universidad del Quindío, Facultad de Ciencias Básicas y Tecnologías \\ Armenia, Quindío - Colombia \\ ${ }^{2}$ Universidade Federal de Mato Grosso, Instituto de Ciências Exatas e da Terra \\ Pontal do Araguaia - MT - Brasil \\ ${ }^{3}$ On leave from Jawaharlal Nehru Technological University \\ Hyderabad-500 085, India \\ E-mails: paulocct@uniquindio.edu.co / jocirei@ufmt.br / vshrao@jntuh.ac.in
}

\begin{abstract}
In this paper we study the influence of weak Allee effect in a predator-prey system model. This effect is included in the prey equation and we determine conditions for the occurrence of Hopf bifurcation. The stability properties of the system and the biological issues of the memory and Allee models on the coexistence of the two species are studied. Finally we present some simulations which allow one to verify the analytical conclusions obtained.
\end{abstract}

Mathematical subject classification: Primary: 34C25; Secondary: 92B05.

Key words: weak Allee effect, population dynamics, Hopf bifurcation, predator-prey model, distributed delay.

\#CAM-302/10. Received: 01/XII/10. Accepted: 19/IV/11.

* Research supported by Master program of Biomathematics, UNIQUINDIO, Colombia

** Research supported by FAPEMAT - Fundação de Amparo à Pesquisa do Estado de Mato Grosso, Grant Number 462997/2009.

*** Research supported by a grant from the Foundation for Scientific Research and Technological Innovation - A division of Sri Vadrevu Seshagiri Rao Memorial Charitable Trust, Hyderabad India. 


\section{Introduction}

It is well known that in nature some species often co-operate amongst themselves in their search for food or when they try to escape from predators. Allee $[15,16]$, has studied extensively the aspects of aggregation and associated co-operative and social characteristics among the members of a species. In population biology Allee effect refers to a population that has a maximal per capita growth rate at intermediate density. This occurs when the per capita growth rate increases with the increase in the density and decreases when the density passes through a critical value. Clearly this situation is different from the logistic growth in which the per capita growth rate is a decreasing function of the density. Even more, when considering coexistence for a single prey species with one or more predator species, then the Allee effect zone is modified, because it depends also on the functional response (see in [6]).

The Allee effect is called weak if there exists no critical density population, below which the per capita rate becomes negative. Taking into account the carrying capacity of the environment with respect to the prey in the per capita growth rate of the population, the weak Allee effect has been modeled in [4] by the following differential equation

$$
\dot{N}(t)=\frac{\varepsilon}{K} N^{2}(t)\left(1-\frac{N(t)}{K}\right)
$$

in which $N(t)$ denotes the prey population at time $t, 0<\varepsilon<1$ is the per capita growth rate of the population and $K>0$ is the carrying capacity of the environment.

In this study we propose and analyze the following system of equations

$$
\begin{aligned}
& \dot{N}(t)=\frac{4 \varepsilon}{K} N^{2}(t)\left(1-\frac{N(t)}{K}\right)-\alpha N(t) P(t) \\
& \dot{P}(t)=-\gamma P(t)+a \beta P(t) \int_{-\infty}^{t} N(\tau) \exp (-a(t-\tau)) d \tau
\end{aligned}
$$

as a model to describe the dynamical behavior of the predator-prey system incorporating a weak Allee effect in the prey populations $N(t)$ and a distributed delay in the predator populations $P(t)$, at time $t$. The parameters $K, \varepsilon, \alpha, \gamma$, $\beta$ and $a$ are positive. $K$ represents the carrying capacity of the environment 
with respect to the prey, $\varepsilon$ is the intrinsic growth rate of the prey, $\alpha$ is the rate of predation, $\gamma$ is the predator mortality in the absence of prey, $\beta$ is the conversion rate and $a$ is the delay parameter. Since $\varepsilon$ is small, we have considered a magnification of $\varepsilon$ by denoting it as $4 \varepsilon$ in the model equations (2) and in the subsequent simulations. This magnification renders clarity to the simulations carried in this paper.

It is well known in biology that group defence helps decrease (or even prevent) the predation due to the enrichment in the ability of the prey to defend or escape from the predators. In view of the considerations in Freedman and Wolkowicz [11], the model equations (2) may also be viewed as a predatorprey system with group defence exhibited by the prey.

The models considered in earlier studies $([1,2,3,5,7,8,10,12,14])$ though similar, but our model differs from those in terms of incorporating weak Allee effect as opposed to a logistic functional response in the prey dynamics. Though our study in this paper allows one to replace the density function $a e^{-a t}$ by a more general function $G:[0, \infty) \rightarrow[0, \infty)$, that satisfies $G(t) \geq 0$ and $\int_{0}^{\infty} G(t) d t=1$, we prefer to retain the second equation in the system (2) in its present form, as no new ideas would be introduced by this replacement.

The present paper is organized as follows: In Section 2, we determine the equilibria and present the local stability of the equilibrium points that do not depend on the parameters of the system. The conditions for occurrence of Hopf bifurcation for the parameter dependent equilibrium and the related analysis are discussed in Section 3. The simulation results are carried using Maple 9 and are described in Section 4. A pseudo Maple code that facilitates the simulations is presented in Section 6. A discussion follows in the final section.

\section{Equilibria and the linear analysis}

In order to determine the equilibria for the system (2), we rewrite (2) in the following form

$$
\begin{aligned}
\dot{N}(t) & =\frac{4 \varepsilon}{K} N^{2}(t)\left(1-\frac{N(t)}{K}\right)-\alpha N(t) P(t) \\
\dot{P}(t) & =-\gamma P(t)+\beta P(t) Q(t) \\
\dot{Q}(t) & =a(N(t)-Q(t)),
\end{aligned}
$$


in which

$$
Q(t)=a \int_{-\infty}^{t} N(\tau) \exp (-a(t-\tau)) d \tau .
$$

The change of variables,

$$
N=K n, \quad P=K p, \quad Q=K q \text { and } t=\frac{s}{\varepsilon},
$$

transforms the system (3) into

$$
\mathbf{x}^{\prime}=f(\mathbf{x}, K)=\left(4 n^{2}(1-n)-\frac{\alpha K}{\varepsilon} n p,-\frac{\gamma}{\varepsilon} p+\frac{\beta K}{\varepsilon} p q, \frac{a}{\varepsilon}(n-q)\right),
$$

where the prime represents the derivative respect to $s, \mathbf{x}=(n, p, q) \in \mathbb{R}^{3}$ and $K \in(0, \infty)$. It is easy to see that system (4) has the following equilibria

$$
\begin{aligned}
\left(n_{1}, p_{1}, q_{1}\right) & =(0,0,0),\left(n_{2}, p_{2}, q_{2}\right)=(1,0,1) \text { and } \\
\left(n_{3}, p_{3}, q_{3}\right) & =\left(\frac{\gamma}{\beta K}, \frac{4 \varepsilon \gamma(\beta K-\gamma)}{\alpha \beta^{2} K^{3}}, \frac{\gamma}{\beta K}\right) .
\end{aligned}
$$

The following result provides sufficient conditions for the local stability and equilibria $\left(n_{1}, p_{1}, q_{1}\right)$ and $\left(n_{2}, p_{2}, q_{2}\right)$.

Proposition 2.1. For the system (4) the equilibrium $\left(n_{1}, p_{1}, q_{1}\right)$ is unstable for all $K>0$. On the other hand, the equilibrium $\left(n_{2}, p_{2}, q_{2}\right)$ is locally asymptotically stable if $K<\frac{\gamma}{\beta}$ and unstable if $K>\frac{\gamma}{\beta}$.

Proof. We establish this result by considering the Jacobian matrix of system (4), which may be written as

$$
\mathbf{J}(n, p, q)=\left[\begin{array}{ccc}
\frac{-12 \varepsilon n^{2}+8 \varepsilon n-\alpha K p}{\varepsilon} & \frac{-\alpha K}{\varepsilon} n & 0 \\
0 & \frac{-\gamma+\beta K q}{\varepsilon} & \frac{\beta K}{\varepsilon} p \\
\frac{a}{\varepsilon} & 0 & -\frac{a}{\varepsilon}
\end{array}\right] .
$$

The Jacobian matrix $\mathbf{J}\left(n_{1}, p_{1}, q_{1}\right)$ has eigenvalues $0,-\frac{\gamma}{\varepsilon}$ and $-\frac{a}{\varepsilon}$ and by reduction procedure to center manifold ([13]), we obtain $\dot{n}=4 n^{2}$ and from this it follows that the equilibrium $\left(n_{1}, p_{1}, q_{1}\right)$ is unstable for all $K>0$. 
Similarly the eigenvalues associated with $\mathbf{J}\left(n_{2}, p_{2}, q_{2}\right)$ are given by -1 , $-\frac{a}{\varepsilon}$ and $\frac{-\gamma+\beta K}{\varepsilon}$. Thus $\left(n_{2}, p_{2}, q_{2}\right)$ is locally asymptotically stable provided $K<\frac{\gamma}{\beta}$ and unstable when $K>\frac{\gamma}{\beta}$.

Remark 2.2. The instability of the origin $\left(n_{1}, p_{1}, q_{1}\right)$ may be interpreted as the non vanishing of the species simultaneously in increasing time. On the other hand the instability of the equilibrium $\left(n_{2}, p_{2}, q_{2}\right)$ implies that the carrying capacity of the environment $K$ and the conversion rate $\beta$ of the predators do not support the predator population which vanishes in time. This conclusion is the same as observed in ([9]). The equilibrium $\left(\frac{\gamma}{\beta K}, \frac{4 \varepsilon \gamma(\beta K-\gamma)}{\alpha \beta^{2} K^{3}}, \frac{\gamma}{\beta K}\right)$ is biologically meaningful only if

$$
\beta K-\gamma>0 \text { or } \frac{\gamma}{\beta K}<1 .
$$

The condition (6) means that the dependence on time of the predator growth rate is positive, at least when $Q$ assumes the value $K$ (see [9]). Moreover, $0<\frac{\gamma}{\beta K}<1$ implies $0<\frac{\gamma}{K}<\beta$, provided that the mortality rate $\gamma$ of the predator species with respect to the maximum capacity of prey $K$, must be less than the conversion rate $\beta$. However, for large enough $K$, it is always true and necessarily (for small populations of prey), this does not ensure the survival of the predator species.

We shall discuss the stability of this equilibrium in the next section.

\section{Stability analysis of the equilibrium $\left(n_{3}, p_{3}, q_{3}\right)$}

In this section we study the stability properties of the equilibrium $\left(n_{3}, p_{3}, q_{3}\right)=$ $\left(\frac{\gamma}{\beta K}, \frac{4 \varepsilon \gamma(\beta K-\gamma)}{\alpha \beta^{2} K^{3}}, \frac{\gamma}{\beta K}\right)$. We study the local codimension one Hopf bifurcation which occurs in the system (4) and also determine the direction of the bifurcation.

The Jacobian matrix in $\left(n_{3}, p_{3}, q_{3}\right)$ is given by

$$
\mathbf{J}\left(n_{3}, p_{3}, q_{3}\right)=\left[\begin{array}{ccc}
\frac{4 \gamma(\beta K-2 \gamma)}{\beta^{2} K^{2}} & -\frac{\alpha \gamma}{\beta \varepsilon} & 0 \\
0 & 0 & \frac{4 \gamma(\beta K-\gamma)}{\alpha \beta K^{2}} \\
\frac{a}{\varepsilon} & 0 & -\frac{a}{\varepsilon}
\end{array}\right]
$$


and the characteristic equation associated with $\mathbf{J}\left(n_{3}, p_{3}, q_{3}\right)$ is

$$
\begin{gathered}
\lambda^{3}+\frac{a \beta^{2} K^{2}+4 \gamma \varepsilon(2 \gamma-\beta K)}{\beta^{2} \varepsilon K^{2}} \lambda^{2} \\
+\frac{4 a \gamma(2 \gamma-\beta K)}{\beta^{2} \varepsilon K^{2}} \lambda+\frac{4 a \gamma^{2}(\beta K-\gamma)}{\beta^{2} \varepsilon^{2} K^{2}}=0 .
\end{gathered}
$$

The following proposition is a direct consequence of the Routh-Hurwitz stability criterion (see [8]).

Proposition 3.1. The critical point $\left(n_{3}, p_{3}, q_{3}\right)$ is locally asymptotically stable for the system (4) if the following inequalities are satisfied

$$
\begin{gathered}
\beta K-\gamma>0 \\
2 \gamma-\beta K>0 \\
\frac{a \beta^{2} K^{2}+4 \gamma \varepsilon(2 \gamma-\beta K)}{\beta^{2} \varepsilon K^{2}} \times \frac{4 a \gamma(2 \gamma-\beta K)}{\beta^{2} \varepsilon K^{2}}-\frac{4 a \gamma^{2}(\beta K-\gamma)}{\beta^{2} \varepsilon^{2} K^{2}}>0 .
\end{gathered}
$$

Remark 3.2. The condition (8) coincide with (6). The same is observed in [8]. From (8) and (9), it follows that

$$
\frac{\beta}{2}<\frac{\gamma}{K}<\beta
$$

Clearly this inequality highlights the contribution of the weak Allee effect, i.e. the refinement in the relationship between $\beta, \gamma$ and $K$.

A rearrangement of terms in (10) yields

$$
a>\frac{\gamma\left(16 \beta \gamma \varepsilon K-16 \gamma^{2} \varepsilon-4 \beta^{2} \varepsilon K^{2}+\beta^{3} K^{3}-\beta^{2} \gamma K^{2}\right)}{\beta^{2} K^{2}(2 \gamma-\beta K)} .
$$

and this inequality is trivially satisfied if the right hand side of (11) is either zero or negative, in which case the equilibrium $\left(n_{3}, p_{3}, q_{3}\right)$ is locally asymptotically stable for all $a>0$. The case of interest for us would be when

$$
a_{0}=\frac{\gamma\left(16 \beta \gamma \varepsilon K-16 \gamma^{2} \varepsilon-4 \beta^{2} \varepsilon K^{2}+\beta^{3} K^{3}-\beta^{2} \gamma K^{2}\right)}{\beta^{2} K^{2}(2 \gamma-\beta K)}>0 .
$$




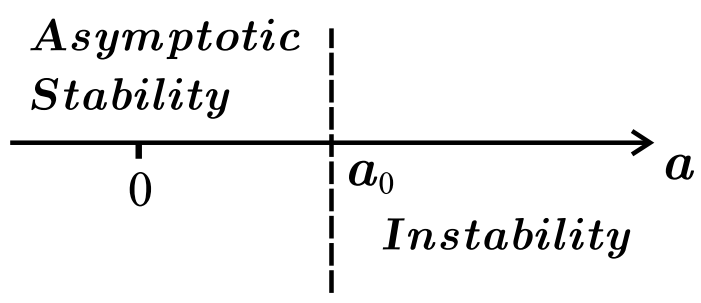

Figure 1 - Critical value of the bifurcation parameter $a$.

In this case, $\left(n_{3}, p_{3}, q_{3}\right)$ loses its stability at $a=a_{0}$ (see Fig. 1$)$. We study the local codimension one Hopf bifurcation which occurs in the system (4) for the state variables $(n, p, q) \in \mathbb{R}^{3}$ and the parameter $a \in \mathbb{R}_{+}$.

To simplify the calculations we introduce the notation $b=\frac{1}{\beta K}$ and rewrite conditions (8)-(10) respectively as

$$
\begin{gathered}
\frac{1}{2}<\gamma b<1 \\
16 \varepsilon \gamma^{2} b^{3}+4 \varepsilon b-16 \varepsilon \gamma b^{2}-1+\gamma b<0 \\
a_{0}=\frac{\gamma}{1-2 \gamma b}\left(16 \varepsilon \gamma^{2} b^{3}+4 \varepsilon b-16 \varepsilon \gamma b^{2}-1+\gamma b\right) .
\end{gathered}
$$

At $a=a_{0}$ the eigenvalues of system (4) are given by

$$
\lambda_{0}\left(a_{0}\right)=\frac{\gamma(\gamma b-1)}{\varepsilon(2 \gamma b-1)}<0, \quad \lambda_{1,2}\left(a_{0}\right)= \pm i \omega
$$

in which

$$
\omega=\frac{2 \gamma}{\varepsilon} \sqrt{\varepsilon b\left(-16 \varepsilon \gamma^{2} b^{3}-4 \varepsilon b+16 \varepsilon \gamma b^{2}+1-\gamma b\right)} .
$$

We have the following result

Theorem 3.3. With the notation and conditions (13)-(17) the real part of the eigenvalue $\lambda_{1}(a)$ that is $\operatorname{Re}(\lambda(a))$ satisfies the conditions that

$$
\frac{d}{d \lambda}\left(\operatorname{Re}\left(\lambda_{1}\left(a_{0}\right)\right)\right) \neq 0
$$


Proof. Note that $\lambda_{1}\left(a_{0}\right)=i \omega$ and define the function

$$
\begin{aligned}
F(\lambda, a)= & \lambda^{3}+\frac{a-4 \varepsilon \gamma b+8 \varepsilon \gamma^{2} b^{2}}{\varepsilon} \lambda^{2}+\frac{4 a \gamma b(2 \gamma b-1)}{\varepsilon} \lambda \\
& +\frac{4 a \gamma^{2} b(1-\gamma b)}{\varepsilon^{2}}
\end{aligned}
$$

obtained from the right hand side of (7) in which $b=\frac{1}{\beta K}$.

Since $F\left(\lambda_{1}\left(a_{0}\right), a_{0}\right)=F\left(i \omega, a_{0}\right)=0$ and $i \omega$ is a simple root of $F\left(\lambda, a_{0}\right)$; by the implicit function theorem exist a unique one $\lambda=\lambda(a)$ in a neighborhood of $a_{0}$ such that

$$
\lambda_{1}^{\prime}\left(a_{0}\right)=-\frac{F_{a}^{\prime}\left(i \omega, a_{0}\right)}{F_{\lambda}^{\prime}\left(i \omega, a_{0}\right)},
$$

where

$$
F_{a}^{\prime}(\lambda, a)=\frac{\lambda^{2}}{\varepsilon}+\frac{4 \gamma b(2 \gamma b-1)}{\varepsilon} \lambda+\frac{4 \gamma^{2} b(1-\gamma b)}{\varepsilon^{2}}
$$

and

$$
F_{\lambda}^{\prime}(\lambda, a)=3 \lambda^{2}+\frac{2\left(a-4 \varepsilon \gamma b+8 \varepsilon \gamma^{2} b^{2}\right)}{\varepsilon} \lambda+\frac{4 a \gamma b(2 \gamma b-1)}{\varepsilon} .
$$

Thus

$$
F_{a}^{\prime}\left(i \omega, a_{0}\right)=(2 \gamma b-1)\left(-\omega^{2} \varepsilon-4 i \omega \varepsilon \gamma b+8 i \omega \varepsilon \gamma^{2} b^{2}+4 \gamma^{2} b-4 \gamma^{3} b^{2}\right)
$$

and

$$
\begin{aligned}
F_{\lambda}^{\prime}\left(i \omega, a_{0}\right)= & \varepsilon\left(-3 \omega^{2} \varepsilon+6 \omega^{2} \varepsilon \gamma b-2 i \omega \gamma+2 i \omega \gamma^{2} b-192 \varepsilon \gamma^{4} b^{4}\right. \\
& +128 \varepsilon \gamma^{5} b^{5}-16 \varepsilon \gamma^{2} b^{2}+96 \varepsilon \gamma^{3} b^{3}+4 \gamma^{2} b \\
& \left.-12 \gamma^{3} b^{2}+8 \gamma^{4} b^{3}\right) .
\end{aligned}
$$

Since

$$
\frac{d}{d a}\left(\operatorname{Re}\left(\lambda_{1}\left(a_{0}\right)\right)\right)=\operatorname{Re}\left(\frac{d}{d a}\left(\lambda_{1}\left(a_{0}\right)\right)\right),
$$

substituting

$$
\omega^{2}=\frac{4 \gamma^{2} b}{\varepsilon}\left(-16 \varepsilon \gamma^{2} b^{3}-4 \varepsilon b+16 \varepsilon \gamma b^{2}+1-\gamma b\right)
$$


in $\operatorname{Re}\left(\frac{d}{d a}\left(\lambda_{1}\left(a_{0}\right)\right)\right)$ we have

$\operatorname{Re}\left(\frac{d}{d a}\left(\lambda_{1}\left(a_{0}\right)\right)\right)=\frac{-2 b(2 \gamma b-1)^{2}\left(16 \varepsilon \gamma^{2} b^{3}+4 \varepsilon b-16 \varepsilon \gamma b^{2}-1+\gamma b\right)}{G(\varepsilon, \cdot)}$,

where

$$
\begin{aligned}
G(\varepsilon, \cdot)= & 256 \varepsilon^{2} \gamma^{4} b^{6}-512 \varepsilon^{2} \gamma^{3} b^{5}+16 \varepsilon \gamma^{3} b^{4}+384 \varepsilon^{2} \gamma^{2} b^{4}-32 \varepsilon \gamma^{2} b^{3} \\
& -\gamma^{2} b^{2}-128 \varepsilon^{2} \gamma b^{3}+20 \varepsilon \gamma b^{2}+2 \gamma b+16 \varepsilon^{2} b^{2}-4 \varepsilon b-1
\end{aligned}
$$

From (14) the numerator in the right hand side of (19) is positive. To complete the proof we need to show that $G(\varepsilon, \cdot) \neq 0$. To this end, consider the equation $G(\epsilon)=0$, and observe that

$$
\varepsilon_{1}=\frac{(\sqrt{5}-1)(\gamma b-1)}{8 b(2 \gamma b-1)^{2}}<0 \text { and } \varepsilon_{2}=\frac{(\sqrt{5}+1)(1-\gamma b)}{8 b(2 \gamma b-1)^{2}}>0,
$$

are its roots. Since $G^{\prime \prime}(\varepsilon, \cdot)=32 b^{2}(2 \gamma b-1)^{4}>0, G$ has a minimum in the interval $\left(\varepsilon_{1}, \varepsilon_{2}\right)$. Rewriting (14) as

$$
0<\varepsilon<\frac{1-\gamma b}{4 b(2 \gamma b-1)^{2}}
$$

we have

$$
0<\varepsilon<\frac{1-\gamma b}{4 b(2 \gamma b-1)^{2}}<\frac{(\sqrt{5}+1)(1-\gamma b)}{8 b(2 \gamma b-1)^{2}}=\varepsilon_{2} .
$$

So, $G(\varepsilon, \cdot)<0$ which implies $\operatorname{Re}\left(\frac{d}{d a}\left[\lambda_{1}\left(a_{0}\right)\right]\right)<0$, and this proves the Theorem.

Now we introduce the new bifurcation parameter $\mu$ by

$$
a(\mu)=\frac{a_{0}}{1+a_{0} \mu}
$$

(see Fig. 1). In this case, the equilibrium $\left(n_{3}, p_{3}, q_{3}\right)$ is locally asymptotically stable for $\mu<0$ and looses its stability at $\mu=0$, that is, $\mu=0$ is the new 
bifurcation point. Further,

$$
\begin{gathered}
\left.\operatorname{Re}\left(\frac{d}{d \mu}\left[\lambda_{1}(a(\mu))\right]\right)\right|_{\mu=0} \\
=\frac{2 \gamma^{2} b\left(16 \varepsilon \gamma^{2} b^{3}-16 \varepsilon \gamma b^{2}+\gamma b+4 \varepsilon b-1\right)^{3}}{G(\varepsilon, \cdot)}>0 .
\end{gathered}
$$

\subsection{Projection to the center manifold}

At beginning of this section we show that the eigenvalues curve of system (4) satisfies the transversality conditions of the Hopf's Theorem. In the following, we will restrict system (4) to the center manifold, that is, we will project the vector field associated to system (4) to the center manifold. So, the ODE system given by (4) will behave as a system on plane.

To reach this purpose, we consider the change of variables $b=\frac{1}{\beta K}$ to convert the equilibrium $\left(n_{3}, p_{3}, q_{3}\right)$ to the form $\left(\gamma b, \frac{4 \varepsilon \gamma b(1-\gamma b)}{\alpha K}, \gamma b\right)$. Furthermore, we move this equilibrium to origin introducing the variables

$$
x=n-\gamma b, \quad y=p-\frac{4 \varepsilon \gamma b(1-\gamma b)}{\alpha K}, \quad z=q-\gamma b .
$$

Thus, with the new variables we carried out system (4) to the equivalent system

$$
\begin{aligned}
& \dot{x}=4(x+\gamma b)^{2}(1-(x+\gamma b))-\frac{\alpha K}{\varepsilon}(x+\gamma b)\left(y+\frac{4 \varepsilon \gamma b(1-\gamma b)}{\alpha K}\right) \\
& \dot{y}=\frac{-\gamma}{\varepsilon}\left(y+\frac{4 \varepsilon \gamma b(1-\gamma b)}{\alpha K}\right)+\frac{1}{\varepsilon b}\left(y+\frac{4 \varepsilon \gamma b(1-\gamma b)}{\alpha K}\right)(z+\gamma b) \\
& \dot{z}=\frac{a_{0}}{\varepsilon\left(1+a_{0} \mu\right)}(x+\gamma b-(z+\gamma b))
\end{aligned}
$$

At $\mu=0$ (i.e. at $a=a_{0}$ ) we have

$$
\begin{aligned}
& \dot{x}=-4 x^{3}-12 \gamma b x^{2}+4 x^{2}-8 \gamma^{2} b^{2} x+4 \gamma b x-\frac{\alpha K}{\varepsilon} x y-\frac{\alpha K \gamma b}{\varepsilon} y \\
& \dot{y}=\frac{4 \gamma}{\alpha K} z-\frac{4 \gamma^{2} b}{\alpha K} z+\frac{1}{\varepsilon b} y z \\
& \dot{z}=\frac{\omega^{2}}{4 \gamma b(2 \gamma b-1)}(x-z) .
\end{aligned}
$$


The eigenvectors of the above system associated with the eigenvalues $\lambda_{0}\left(a_{0}\right)=\frac{\gamma(\gamma b-1)}{\varepsilon(2 \gamma b-1)}$ and $\lambda_{1,2}\left(a_{0}\right)= \pm i \omega$ are

$$
s_{0}=\left[\begin{array}{c}
\frac{4 \gamma^{2} b^{2}(2 \gamma b-1)}{\omega^{2}} \\
\frac{\varepsilon}{\alpha K} \\
\frac{-1}{4(2 \gamma b-1)}
\end{array}\right] \text { and } s_{1,2}=\left[\begin{array}{c}
1 \\
0 \\
1
\end{array}\right] \pm i\left[\begin{array}{c}
\frac{4 \gamma b(2 \gamma b-1)}{\omega} \\
\frac{4 \gamma(\gamma b-1)}{\alpha \omega K} \\
0
\end{array}\right]
$$

respectively.

We take $s_{0}$ and the real and imaginary parts of $s_{1,2}$ as a new basis for $\mathbb{R}^{3}$. So, with the change of coordinate

$$
\left[\begin{array}{l}
x \\
y \\
z
\end{array}\right]=A\left[\begin{array}{l}
x_{1} \\
x_{2} \\
x_{3}
\end{array}\right]
$$

where

$$
A=\left[\begin{array}{ccc}
1 & \frac{4 \gamma b(2 \gamma b-1)}{\omega} & \frac{4 \gamma^{2} b^{2}(2 \gamma b-1)}{\omega^{2}} \\
0 & \frac{4 \gamma(\gamma b-1)}{\omega \alpha K} & \frac{-\varepsilon}{\alpha K} \\
1 & 0 & \frac{-1}{4(2 \gamma b-1)}
\end{array}\right]
$$

we transform system (22) to an equivalent system

$$
\begin{aligned}
& \dot{x}_{1}=\omega x_{2}+U_{1,2}(\mathbf{x})+U_{1,3}(\mathbf{x}) \\
& \dot{x}_{2}=-\omega x_{1}+U_{2,2}(\mathbf{x})+U_{2,3}(\mathbf{x}) \\
& \dot{x}_{3}=\frac{\gamma(\gamma b-1)}{\varepsilon(2 \gamma b-1)} x_{1}+U_{3,2}(\mathbf{x})+U_{3,3}(\mathbf{x}),
\end{aligned}
$$

for $\mathbf{x}=\left(x_{1}, x_{2}, x_{3}\right)$ and

$$
\begin{aligned}
U_{1,2}(\mathbf{x})= & -\frac{4 \omega^{2}}{D}(\gamma b-1)(3 \gamma b-1) x_{1}^{2} \\
& -\frac{16 \gamma^{2} b}{\varepsilon D}(\gamma b-1)(2 \gamma b-1)\left(24 \varepsilon \gamma^{2} b^{3}-20 \varepsilon \gamma b^{2}+\gamma b\right.
\end{aligned}
$$




$$
\begin{aligned}
& +4 \varepsilon b-1) x_{2}^{2}+\frac{\gamma^{2} b}{4 \omega^{2} \varepsilon^{2} D}\left(16 \varepsilon \gamma^{3} b^{3}+4 \gamma^{3} b^{3}-4 \gamma^{2} b\right. \\
& \left.-7 \omega^{2} \varepsilon \gamma b-4 \omega^{2} \varepsilon^{2} b+8 \omega^{2} \varepsilon+3 \varepsilon D\right) x_{3}^{2} \\
& -\frac{\omega}{\varepsilon \gamma b D}(\gamma b-1)\left(48 \varepsilon \gamma^{2} b^{3}-40 \varepsilon \gamma b^{2}+3 \gamma b\right. \\
& +8 \varepsilon b-2) x_{1} x_{2}+\frac{4 \gamma^{2} b}{\varepsilon D}\left(8 \varepsilon \gamma^{2} b^{3}+3 \gamma^{2} b^{2}-4 \varepsilon \gamma b^{2}\right. \\
& -5 \gamma b+2) x_{1} x_{3}-\frac{4 \gamma b}{\omega \varepsilon^{2} D}(\gamma b-1)\left(4 \varepsilon \gamma^{3} b^{2}+\gamma^{3} b\right. \\
& \left.-4 \omega^{2} \varepsilon^{2} \gamma b-\gamma^{2}+\varepsilon^{2} \omega^{2}\right) x_{2} x_{3}
\end{aligned}
$$

$$
\begin{aligned}
U_{1,3}(\mathbf{x})= & -\frac{4 \omega^{2}}{D}(\gamma b-1) x_{1}^{3}-\frac{256 \gamma^{3} b^{3}}{\omega D}(\gamma b-1)(2 \gamma b-1)^{3} x_{2}^{3} \\
& -\frac{256 \gamma^{6} b^{6}}{\omega^{4} D}(\gamma b-1)(2 \gamma b-1)^{3} x_{3}^{3} \\
& -\frac{48 \omega \gamma b}{D}(\gamma b-1)(2 \gamma b-1) x_{1}^{2} x_{2} \\
& -\frac{48 \gamma^{2} b^{2}}{D}(\gamma b-1)(2 \gamma b-1) x_{1}^{2} x_{3} \\
& -\frac{192 \gamma^{2} b^{2}}{D}(\gamma b-1)(2 \gamma b-1)^{2} x_{1} x_{2}^{2} \\
& -\frac{768 \gamma^{4} b^{4}}{\omega^{2} D}(\gamma b-1)(2 \gamma b-1)^{3} x_{2}^{2} x_{3} \\
& -\frac{192 \gamma^{4} b^{4}}{\omega^{2} D}(\gamma b-1)(2 \gamma b-1)^{2} x_{1} x_{3}^{2} \\
& -\frac{768 \gamma^{5} b^{5}}{\omega^{3} D}(\gamma b-1)(2 \gamma b-1)^{3} x_{2} x_{3}^{2} \\
& -\frac{384 \gamma^{3} b^{3}}{\omega D}(\gamma b-1)(2 \gamma b-1) x_{1} x_{2} x_{3}
\end{aligned}
$$

$$
U_{2,2}(\mathbf{x})=\frac{4 \omega^{3} \varepsilon}{D}(2 \gamma b-1)(3 \gamma b-1) x_{1}^{2}
$$




$$
\begin{aligned}
& +\frac{16 \omega \gamma b}{D}(2 \gamma b-1)^{2}\left(24 \varepsilon \gamma^{2} b^{3}-20 \varepsilon \gamma b^{2}+\gamma b+4 \varepsilon b-1\right) x_{2}^{2} \\
& -\frac{\gamma}{16 \omega \varepsilon^{2} D(2 \gamma b-1)}\left(28 \gamma^{4} b^{3}-60 \gamma^{3} b^{2}-16 \varepsilon \gamma^{2} b^{2}\right. \\
& +16 \omega^{2} \varepsilon^{2} \gamma b^{2}-16 \varepsilon^{2} \gamma b^{2} D+32 \gamma^{2} b-4 \omega^{2} \varepsilon^{2} b \\
& \left.+3 \varepsilon D-\omega^{2} \varepsilon\right) x_{3}^{2}-\frac{1}{2 \varepsilon^{2} b D}\left(16 \varepsilon \gamma^{3} b^{3}+8 \omega^{2} \varepsilon^{2} \gamma b^{2}+4 \gamma^{3} b^{2}\right. \\
& +48 \varepsilon^{2} \gamma b^{2} D-4 \gamma^{2} b-3 \omega^{2} \varepsilon \gamma b-4 \omega^{2} \varepsilon^{2} b-16 \varepsilon^{2} b D \\
& \left.+4 \omega^{2} \varepsilon+\varepsilon D\right) x_{1} x_{2}+\frac{\omega}{8 \varepsilon \gamma b D}\left(64 \varepsilon \gamma^{3} b^{3}+4 \gamma^{3} b^{2}-16 \varepsilon \gamma^{2} b^{2}\right. \\
& \left.-32 \omega^{2} \varepsilon^{2} \gamma b^{2}-4 \gamma^{2} b+8 \omega^{2} \varepsilon^{2} b+3 \omega^{2} \varepsilon\right) x_{1} x_{3} \\
& -\frac{1}{8 \varepsilon^{2} b D(2 \gamma b-1)}\left(16 \gamma^{4} b^{3}-36 \gamma^{3} b^{2}-16 \varepsilon \gamma^{2} b^{2}-32 \varepsilon^{2} \gamma b^{2} D\right. \\
& \left.+8 \omega^{2} \varepsilon^{2} \gamma b^{2}+20 \gamma^{2} b+4 \omega^{2} \varepsilon \gamma b+8 \varepsilon^{2} b D+2 \varepsilon D-5 \omega^{2} \varepsilon\right) x_{2} x_{3}, \\
& U_{2,3}(\mathbf{x})=\frac{4 \omega^{3} \varepsilon}{\gamma D}(2 \gamma b-1) x_{1}^{3}+\frac{256 \varepsilon \gamma^{2} b^{3}}{D}(2 \gamma b-1)^{4} x_{2}^{3} \\
& +\frac{256 \varepsilon \gamma^{5} b^{6}}{\omega^{3} D}(2 \gamma b-1)^{4} x_{3}^{3}+\frac{48 \omega^{2} b}{D}(2 \gamma b-1)^{2} x_{1}^{2} x_{2} \\
& +\frac{48 \omega \varepsilon \gamma b^{2}}{D}(2 \gamma b-1)^{2} x_{1}^{2} x_{3} \\
& +\frac{192 \omega \varepsilon \gamma b^{2}}{D}(2 \gamma b-1)^{3} x_{1} x_{2}^{2}+\frac{768 \varepsilon \gamma^{3} b^{4}}{\omega D}(2 \gamma b-1)^{4} x_{2}^{2} x_{3} \\
& +\frac{192 \varepsilon \gamma^{3} b^{4}}{\omega D}(2 \gamma b-1)^{3} x_{1} x_{3}^{2}+\frac{768 \varepsilon \gamma^{4} b^{5}}{\omega^{2} D}(2 \gamma b-1)^{4} x_{2} x_{3}^{2} \\
& +\frac{384 \varepsilon \gamma^{2} b^{3}}{D}(2 \gamma b-1)^{3} x_{1} x_{2} x_{3} \\
& U_{3,2}(\mathbf{x})=-\frac{16 \omega^{2}}{D}(\gamma b-1)(2 \gamma b-1)(3 \gamma b-1) x_{1}^{2} \\
& -\frac{64 \gamma^{2} b}{\varepsilon D}(\gamma b-1)(2 \gamma b-1)^{2}\left(24 \varepsilon \gamma^{2} b^{3}-20 \varepsilon \gamma b^{2}\right.
\end{aligned}
$$




$$
\begin{aligned}
& +\gamma b+4 \varepsilon b-1) x_{2}^{2}+\frac{\gamma^{2} b}{\omega^{2} \varepsilon^{2} D}(2 \gamma b-1)\left(16 \varepsilon \gamma^{3} b^{3}+4 \gamma^{3} b^{2}\right. \\
& \left.-4 \gamma^{2} b-7 \omega^{2} \varepsilon \gamma b-4 \omega^{2} \varepsilon^{2} b+8 \omega^{2} \varepsilon+3 \varepsilon D\right) x_{3}^{2} \\
& -\frac{4 \omega}{\varepsilon \gamma b D}(\gamma b-1)(2 \gamma b-1)\left(32 \varepsilon \gamma^{3} b^{3}-16 \varepsilon \gamma^{2} b^{2}+4 \gamma^{2} b\right. \\
& \left.-3 \omega^{2} \varepsilon\right) x_{1} x_{2}+\frac{2}{\varepsilon D}(2 \gamma b-1)\left(32 \varepsilon \gamma^{3} b^{3}+24 \gamma^{4} b^{3}-16 \varepsilon \gamma^{2} b^{2}\right. \\
& \left.-44 \gamma^{3} b^{2}+20 \gamma^{2} b-\omega^{2} \varepsilon\right) x_{1} x_{3}-\frac{16 \gamma b}{\omega \varepsilon^{2} D}(2 \gamma b-1)\left(4 \varepsilon \gamma^{3} b^{2}\right. \\
& \left.+\gamma^{3} b-4 \omega^{2} \varepsilon^{2} \gamma b-\gamma^{2}+\omega^{2} \varepsilon^{2}\right) x_{2} x_{3}
\end{aligned}
$$

$$
\begin{aligned}
U_{3,3}(\mathbf{x})= & -\frac{16 \omega^{2}}{D}(\gamma b-1)(2 \gamma b-1) x_{1}^{3} \\
& -\frac{1024 \gamma^{3} b^{3}}{\omega D}(\gamma b-1)(2 \gamma b-1)^{4} x_{2}^{3} \\
& -\frac{1024 \gamma^{6} b^{6}}{\omega^{4} D}(\gamma b-1)(2 \gamma b-1)^{4} x_{3}^{3} \\
& -\frac{192 \omega \gamma b}{D}(\gamma b-1)(2 \gamma b-1)^{2} x_{1}^{2} x_{2} \\
& -\frac{192 \gamma^{2} b^{2}}{D}(\gamma b-1)(2 \gamma b-1)^{2} x_{1}^{2} x_{3} \\
& -\frac{768 \gamma^{2} b^{2}}{D}(\gamma b-1)(2 \gamma b-1)^{3} x_{1} x_{2}^{2} \\
& -\frac{3072 \gamma^{4} b^{4}}{\omega D}(\gamma b-1)(2 \gamma b-1)^{4} x_{2}^{2} x_{3} \\
& -\frac{768 \gamma^{4} b^{4}}{\omega^{2} D}(\gamma b-1)(2 \gamma b-1)^{3} x_{1} x_{3}^{2} \\
& -\frac{3072 \gamma^{5} b^{5}}{\omega^{3} D}(\gamma b-1)(2 \gamma b-1)^{4} x_{2} x_{3}^{2} \\
& -\frac{1536 \gamma^{3} b^{3}}{\omega D}(\gamma b-1)(2 \gamma b-1)^{3} x_{1} x_{2} x_{3},
\end{aligned}
$$


with

$$
\begin{aligned}
D= & \omega^{2}\left(-16 \varepsilon \gamma^{2} b^{3}+16 \varepsilon \gamma b^{2}+\gamma b-4 \varepsilon b-1\right) \\
& +16 \gamma^{2} b^{2}(\gamma b-1)(2 \gamma b-1)^{2}=\frac{4 \gamma^{2} b}{\varepsilon} \times G(\varepsilon, \cdot) .
\end{aligned}
$$

Now we project the system (23) to the center manifold $M$ which is characterized by the fact that it is tangent to the $x_{1} x_{2}$ plane at the origin (the eigenspace corresponding to $\lambda_{1,2}= \pm i \omega$ ) and is locally invariant with respect to the flow of system (23). Note that this center manifold can be parameterized by the variables $x_{1}$ and $x_{2}$ in the form $x_{3}=h\left(x_{1}, x_{2}\right)$ where $h: \mathbb{R}^{2} \rightarrow \mathbb{R}$ admits a Taylor expansion of the form

$$
h\left(x_{1}, x_{2}\right)=\frac{1}{2}\left(h_{11} x_{1}^{2}+2 h_{12} x_{1} x_{2}+h_{22} x_{2}^{2}\right)+O\left(\left(\sqrt{x_{1}^{2}+x_{2}^{2}}\right)^{3}\right),
$$

and, the Center Manifold Theorem implies that $h$ must satisfy

$$
0=h(0,0)=h_{x_{1}}^{\prime}(0,0)=h_{x_{2}}^{\prime}(0,0),
$$

where $h \in C^{k}, k \in \mathbb{N}$ with $k>3$.

If $\left(x_{1}(s), x_{2}(s), x_{3}(s)\right)$ is a solution of system (23) near the origin with initial conditions starting on $M$ then the flow of system (23) remains locally on $M$ for all time, that is $x_{3}(s) \equiv h\left(x_{1}(s), x_{2}(s)\right)$. Consequently

$$
\dot{x}_{3}(s)-h_{x_{1}}^{\prime}\left(x_{1}(s), x_{2}(s)\right) \dot{x}_{1}(s)-h_{x_{2}}^{\prime}\left(x_{1}(s), x_{2}(s)\right) \dot{x}_{2}(s) \equiv 0 .
$$

Using system (23) the above identity turns out to be

$$
\begin{aligned}
& \frac{\gamma(\gamma b-1)}{\varepsilon(2 \gamma b-1)} x_{3}+U_{3,2}\left(x_{1}, x_{2}, x_{3}\right)+U_{3,3}\left(x_{1}, x_{2}, x_{3}\right)-h_{x_{1}}^{\prime}\left(x_{1}, x_{2}\right) \\
&-\left(h_{11} x_{1}+h_{12} x_{2}+O\left(\left(\sqrt{x_{1}^{2}+x_{2}^{2}}\right)^{2}\right)\right)\left(\omega x_{2}+U_{1,2}\left(x_{1}, x_{2}, x_{3}\right)\right. \\
&+\left.U_{1,3}\left(x_{1}, x_{2}, x_{3}\right)\right)-\left(h_{12} x_{1}+h_{22} x_{2}+O\left(\left(\sqrt{x_{1}^{2}+x_{2}^{2}}\right)^{2}\right)\right) \\
& \times \quad\left(-\omega x_{1}+U_{2,2}\left(x_{1}, x_{2}, x_{3}\right)+U_{2,3}\left(x_{1}, x_{2}, x_{3}\right)\right) \equiv 0
\end{aligned}
$$


since

$$
x_{3}=h\left(x_{1}, x_{2}\right)=\frac{1}{2}\left(h_{11} x_{1}^{2}+2 h_{12} x_{1} x_{2}+h_{22} x_{2}^{2}\right)+O\left(\left(\sqrt{x_{1}^{2}+x_{2}^{2}}\right)^{3}\right) .
$$

Omitting the terms of third and higher order we obtain

$$
\begin{gathered}
\frac{\gamma(\gamma b-1)}{2 \varepsilon(2 \gamma b-1)}\left(h_{11} x_{1}^{2}+2 h_{12} x_{1} x_{2}+h_{22} x_{2}^{2}\right) \\
-\omega h_{11} x_{1} x_{2}-\omega h_{12} x_{2}^{2}+\omega h_{12} x_{1}^{2}+\omega h_{22} x_{1} x_{2} \equiv 0 .
\end{gathered}
$$

A rearrangement of terms yields

$$
\begin{aligned}
& \left(\frac{\gamma(\gamma b-1)}{2 \varepsilon(2 \gamma b-1)} h_{11}-\frac{16 \omega^{2}}{D}(\gamma b-1)(2 \gamma b-1)(3 \gamma b-1)+\omega h_{12}\right) x_{1}^{2} \\
+ & \left(\frac{\gamma(\gamma b-1)}{2 \varepsilon(2 \gamma b-1)} h_{22}-\frac{64 \gamma^{2} b}{\varepsilon D}(\gamma b-1)(2 \gamma b-1)^{2}\left(4 \varepsilon \gamma b^{2}(2 \gamma b-1)\right.\right. \\
- & \left.\left.\frac{\omega^{2} \varepsilon}{4 \gamma^{2} b}\right)-\omega h_{12}\right) x_{2}^{2}+\left(\frac{\gamma(\gamma b-1)}{\varepsilon(2 \gamma b-1)} h_{12}-\frac{16 \omega \gamma b}{\varepsilon D}(\gamma b-1)(2 \gamma b-1)\right. \\
\times & \left.\left(\gamma b+8 \varepsilon \gamma b^{2}(2 \gamma b-1)-\frac{\omega^{2} \varepsilon}{2 \gamma^{2} b}\right)-\omega h_{11}+\omega h_{22}\right) x_{1} x_{2} \equiv 0 .
\end{aligned}
$$

Equating the coefficient to zero and solving the resulting system we have

$$
\begin{aligned}
h_{11}= & \frac{32 \omega^{2} \varepsilon \gamma b(\gamma b-1)(2 \gamma b-1)^{2}}{D \times F}\left(8 \varepsilon b(2 \gamma b-1)^{2}(4 \gamma b-1)\right. \\
& \left.+3 \gamma^{2} b^{2}-3 \gamma b+1\right) \\
h_{12}= & \frac{-16(\gamma b-1)(2 \gamma b-1)}{D \times F}\left(\varepsilon \left(8 \gamma^{2} b^{2}(\gamma b-1)(2 \gamma b-1)^{2}(4 \gamma b-1)\right.\right. \\
& \left.-D(3 \gamma b-1))-\gamma^{2} b(\gamma b-1)\left(6 \gamma^{2} b^{2}-9 \gamma b+2\right)\right) \\
h_{22}= & \frac{64 \gamma b(\gamma b-1)(2 \gamma b-1)^{3}}{D \times F}\left(\varepsilon \left(D+8 \gamma^{2} b^{2}(2 \gamma b-1)\left(3 \gamma^{2} b^{2}\right.\right.\right. \\
& \left.-3 \gamma b+1))+2 \gamma^{2} b(4 \gamma b-3)(\gamma b-1)\right),
\end{aligned}
$$

where

$$
F=3 \gamma^{4} b^{3}-6 \gamma^{3} b^{2}+3 \gamma^{2} b+\varepsilon D .
$$


Thus,

$$
\begin{aligned}
h\left(x_{1}, x_{2}\right)= & \frac{8(\gamma b-1)(2 \gamma b-1)}{D \times F}\left(2 T \omega^{2} \varepsilon \gamma b(2 \gamma b-1) x_{1}^{2}\right. \\
& \left.-R x_{1} x_{2}+4 \gamma b(2 \gamma b-1)^{2} S x_{2}^{2}\right),
\end{aligned}
$$

where

$$
\begin{aligned}
T= & 8 \varepsilon b(2 \gamma b-1)^{2}(4 \gamma b-1)+3 \gamma^{2} b^{2}-3 \gamma b+1 \\
R= & \varepsilon\left(8 \gamma^{2} b^{2}(\gamma b-1)(2 \gamma b-1)^{2}(4 \gamma b-1)-D(3 \gamma b-1)\right) \\
& -\gamma^{2} b(\gamma b-1) \times\left(6 \gamma^{2} b^{2}-9 \gamma b+2\right) \\
S= & \varepsilon\left(D+8 \gamma^{2} b^{2}(2 \gamma b-1)\left(3 \gamma^{2} b^{2}-3 \gamma b+1\right)\right) \\
& +2 \gamma^{2} b(4 \gamma b-3)(\gamma b-1) .
\end{aligned}
$$

Finally, to get to the restriction of system (23) to the $\mu=0$ section of the center manifold $M$, we consider the new change of coordinates

$$
y_{1}=x_{1}, \quad y_{2}=x_{2}, \quad y_{3}=x_{3}-h\left(x_{1}, x_{2}\right) .
$$

In this new coordinate system, the equation of the $\mu=0$ section is $y_{3}=0$, that is the $\mu=0$ section is the $y_{1}, y_{2}$ plane. By this coordinate transformation, system (23) becomes

$$
\begin{aligned}
\dot{y}_{1}= & \omega y_{2}+U_{1,2}\left(y_{1}, y_{2}, y_{3}+h\left(y_{1}, y_{2}\right)\right)+U_{1,3}\left(y_{1}, y_{2}, y_{3}+h\left(y_{1}, y_{2}\right)\right) \\
\dot{y}_{2}= & -\omega y_{1}+U_{2,2}\left(y_{1}, y_{2}, y_{3}+h\left(y_{1}, y_{2}\right)\right)+U_{2,3}\left(y_{1}, y_{2}, y_{3}+h\left(y_{1}, y_{2}\right)\right) \\
\dot{y}_{3}= & \frac{\gamma(\gamma b-1)}{\varepsilon(2 \gamma b-1)} y_{3}+U_{3,2}\left(y_{1}, y_{2}, y_{3}+h\left(y_{1}, y_{2}\right)\right) \\
& +U_{3,3}\left(y_{1}, y_{2}, y_{3}+h\left(y_{1}, y_{2}\right)\right) .
\end{aligned}
$$

Restricted to the center manifold $M$ we can write $y_{3}=0$ everywhere and taking into that $\dot{y}_{3}=0$. Hence, the system (23) restricted to the center manifold is given by

$$
\begin{aligned}
\dot{y}_{1}= & \omega y_{2}-\frac{4 \omega^{2}}{D}(\gamma b-1)(3 \gamma b-1) y_{1}^{2}-\frac{16 \gamma^{2} b}{\varepsilon D}(\gamma b-1)(2 \gamma b-1) \\
& \times\left(24 \varepsilon \gamma^{2} b^{3}-20 \varepsilon \gamma b^{2}+\gamma b+4 \varepsilon b-1\right) y_{2}^{2}-\frac{4 \omega \gamma}{\varepsilon D}(\gamma b-1)\left(48 \varepsilon \gamma^{2} b^{3}\right.
\end{aligned}
$$




$$
\begin{aligned}
& \left.-40 \varepsilon \gamma b^{2}+3 \gamma b+8 \varepsilon b-2\right) y_{1} y_{2}+\frac{4 \gamma^{2} b}{\varepsilon D}\left(8 \varepsilon \gamma^{2} b^{3}+3 \gamma^{2} b^{2}-4 \varepsilon \gamma b^{2}\right. \\
& -5 \gamma b+2) h\left(y_{1}, y_{2}\right) y_{1}-\frac{4 \gamma b}{\omega \varepsilon^{2} D}(\gamma b-1)\left(4 \varepsilon \gamma^{3} b^{2}+\gamma^{3} b-4 \omega^{2} \varepsilon^{2} \gamma b\right. \\
& \left.-\gamma^{2}+\varepsilon^{2} \omega^{2}\right) h\left(y_{1}, y_{2}\right) y_{2}-\frac{4 \omega^{2}}{D}(\gamma b-1) y_{1}^{3}-\frac{256 \gamma^{3} b^{3}}{\omega D}(\gamma b-1) \\
& \times(2 \gamma b-1)^{3} y_{2}^{3}-\frac{48 \omega \gamma b}{D}(\gamma b-1)(2 \gamma b-1) y_{1}^{2} y_{2}-\frac{192 \gamma^{2} b^{2}}{D} \\
& \times(\gamma b-1)(2 \gamma b-1)^{2} y_{1} y_{2}^{2}+O\left(\left(\sqrt{y_{1}^{2}+y_{2}^{2}}\right)^{4}\right) \\
& \dot{y}_{2}=-\omega y_{1}+\frac{4 \omega^{3} \varepsilon}{D}(2 \gamma b-1)(3 \gamma b-1) y_{1}^{2}+\frac{16 \omega \gamma b}{D}(2 \gamma b-1)^{2} \\
& \times\left(24 \varepsilon \gamma^{2} b^{3}-20 \varepsilon \gamma b^{2}+\gamma b+4 \varepsilon b-1\right) y_{2}^{2}+\frac{1}{\varepsilon b D} \\
& \times\left(4 \omega^{2} \varepsilon b(2 \gamma b-1)\left(48 \varepsilon \gamma^{2} b^{3}-40 \varepsilon \gamma b^{2}+\gamma b+8 \varepsilon b-1\right)\right. \\
& \left.-\frac{4 \gamma^{2} b(\gamma b-1)^{2}}{\varepsilon}\right) y_{1} y_{2}+\frac{\omega}{4 \gamma b D}\left(\omega^{2}\left(-16 \varepsilon \gamma b^{2}+4 \varepsilon b+\frac{3}{2}\right)\right. \\
& \left.+\frac{2 \gamma^{2} b\left(16 \varepsilon \gamma b^{2}+\gamma b-4 \varepsilon b-1\right)}{\varepsilon}\right) h\left(y_{1}, y_{2}\right) y_{1} \\
& +\frac{\gamma^{2}}{\varepsilon^{2} D(2 \gamma b-1)}\left(64 \varepsilon^{3} b^{3}(2 \gamma b-1)^{4}(4 \gamma b-1)\right. \\
& \left.+(\gamma b-1)^{2}\right) h\left(y_{1}, y_{2}\right) y_{2}+\frac{4 \omega^{3} \varepsilon}{\gamma D}(2 \gamma b-1) y_{1}^{3}+\frac{256 \varepsilon \gamma^{2} b^{3}}{D} \\
& \times(2 \gamma b-1)^{4} y_{2}^{3}+\frac{48 \omega^{2} b}{D}(2 \gamma b-1)^{2} y_{1}^{2} y_{2} \\
& +\frac{192 \omega \varepsilon \gamma b^{2}}{D}(2 \gamma b-1)^{3} y_{1} y_{2}^{2}+O\left(\left(\sqrt{y_{1}^{2}+y_{2}^{2}}\right)^{4}\right) \text {. }
\end{aligned}
$$

The calculations may be summarized in the next proposition, which provides the direction of the Hopf bifurcation.

Proposition 3.4. Consider the one-parameter family of differential equations 
(4). The first Lyapunov coefficient associated with the equilibrium $\left(n_{3}, p_{3}, q_{3}\right)$ is given by

$$
\begin{aligned}
G_{4}= & \frac{1}{\omega \varepsilon^{2} \gamma D^{2} F}\left(-1048576 \omega \varepsilon^{6} \gamma^{6} b^{8}(2 \gamma b-1)^{11}(3 \gamma b-1)^{2}\right. \\
& -32768 \varepsilon^{5} \gamma^{6} b^{7}(\gamma b-1) \times(2 \gamma b-1)^{9} \times\left(12 \gamma^{2} b^{2}+232 \omega \gamma^{2} b^{2}\right. \\
& \left.-7 \gamma b-156 \omega \gamma b+26 \omega+1)-4096 \varepsilon^{4} \gamma^{6} b^{6}(\gamma b-1)(2 \gamma b-1)^{7}\right) \\
& \times\left(516 \omega \gamma^{3} b^{3}+44 \gamma^{3} b^{3}-68 \gamma^{2} b^{2}-862 \omega \gamma^{2} b^{2}+418 \omega \gamma b\right. \\
& +27 \gamma b-62 \omega-3)-512 \varepsilon^{3} \gamma^{6} b^{5}(\gamma b-1)(2 \gamma b-1)^{5} \\
& \times\left(720 \omega \gamma^{4} b^{4}-24 \gamma^{4} b^{4}-1804 \omega \gamma^{3} b^{3}+62 \gamma^{3} b^{3}-59 \gamma^{2} b^{2}\right. \\
& \left.+1632 \omega \gamma^{2} b^{2}-632 \omega \gamma b+25 \gamma b+88 \omega-4\right) \\
& -128 \varepsilon^{2} \gamma^{6} b^{4}(\gamma b-1)^{2}(2 \gamma b-1)^{4} \times\left(260 \omega \gamma^{3} b^{3}-22 \gamma^{3} b^{3}\right. \\
& \left.-466 \omega \gamma^{2} b^{2}+43 \gamma^{2} b^{2}+262 \omega \gamma b-25 \gamma b-48 \omega+4\right) \\
& -32 \gamma^{6} b^{3}(\gamma b-1)^{4}(2 \gamma b-1)^{2}\left(44 \omega \gamma^{2} b^{2}+6 \gamma^{2} b^{2}\right. \\
& -38 \omega \gamma b-7 \gamma b+4 \omega+2)+16 \omega \gamma^{6} b^{2}(\gamma b-1)^{4} \\
& \times\left(28 \gamma^{3} b^{3}-47 \gamma^{2} b^{2}+25 \gamma b-4\right) .
\end{aligned}
$$

Proof. Consider the two dimensional ODE system

$$
\begin{aligned}
& \dot{x}=-\omega y+f_{2}(x, y)+f_{3}(x, y) \\
& \dot{y}=\omega x+g_{2}(x, y)+g_{3}(x, y),
\end{aligned}
$$

where

$$
\begin{aligned}
& f_{2}(x, y)=a_{1} x^{2}+a_{2} x y+a_{3} y^{2}, \\
& f_{3}(x, y)=b_{1} x^{3}+b_{2} x^{2} y+b_{3} x y^{2}+b_{4} y^{4}, \\
& g_{2}(x, y)=c_{1} x^{2}+c_{2} x y+c_{3} y^{2} \\
& g_{3}(x, y)=d_{1} x^{3}+d_{2} x^{2} y+d_{3} x y^{2}+d_{4} y^{4} .
\end{aligned}
$$


In accordance with Bautin's Lemma (see [8]) the first Poincaré-Lyapunov coefficient for system (26) is given by

$$
\begin{gathered}
G_{4}=\frac{1}{4}\left(3 b_{1}+b_{3}+d_{2}+3 d_{4}\right)+\frac{1}{4 \omega} \\
\times\left(a_{1} a_{2}+a_{2} a_{3}-c_{1} c_{2}-c_{2} c_{3}+2\left(a_{3} c_{3}-a_{1} c_{1}\right)\right) .
\end{gathered}
$$

Comparing (26) with (24) and using (27), we obtain $G_{4}$ as given by (25).

With the results obtained in this section we can enunciate the following Theorem.

Theorem 3.5. If (12) holds and $G_{4}<0$ (respectively $G_{4}>0$ ), then there exists $a \delta>0$ such that for each $a \in\left(a_{0}-\delta, a_{0}\right)$ (respectively $a \in\left(a_{0}, a_{0}+\right.$ $\delta)$ ), the system (4) (or equivalently system (3)) has a unique orbitally asymptotically stable (respectively unstable) periodic orbit around the equilibrium $\left(n_{3}, p_{3}, q_{3}\right)$.

\section{Simulation results}

In this section we present some simulations carried in Maple 9 to verify the veracity of the analytical results obtained for system (4).

The equilibrium point $(0,0,0)$ is always unstable; to illustrate this case we made the phase portrait in the case by choosing $\varepsilon=0.5, K=1.4, \alpha=0.5$, $\gamma=0.7, \beta=0.8$ y $a=0.25$ (see Fig. 2).

The equilibrium point $(1,0,1)$ is stable if $\beta K-\gamma<0$ and unstable if $\beta K-\gamma>0$. So, to see the stability of this equilibrium we take $\varepsilon=0.5$, $K=1.4, \alpha=0.5, \gamma=0.7, \beta=0.3$ and $a=0.25$ (see Fig. 3-(a)); on the other hand, the instability is obtained when $\varepsilon=0.5, K=1.4, \alpha=0.5$, $\gamma=0.7, \beta=0.8$ and $a=0.25$ (see Fig. 3-(b)).

Finally, we illustrate the stability properties of the equilibrium $\left(n_{3}, p_{3}, q_{3}\right)$. If we consider the following values of the parameters $\varepsilon=0.2, K=1.4$, $\alpha=0.5, \gamma=0.7, \beta=0.8$ and $a=0.985$, the equilibrium $\left(n_{3}, p_{3}, q_{3}\right)$ is asymptotically stable (see Fig. 4-(a)). Note that these values yield $\left(n_{3}, p_{3}, q_{3}\right)=(0.625,0.2678571428,0.625)$ and the conditions (8), (9) and (10) are equivalent to $0.42>0,-0.28<0$ and $a=0.985>a_{0}=0.925$ 


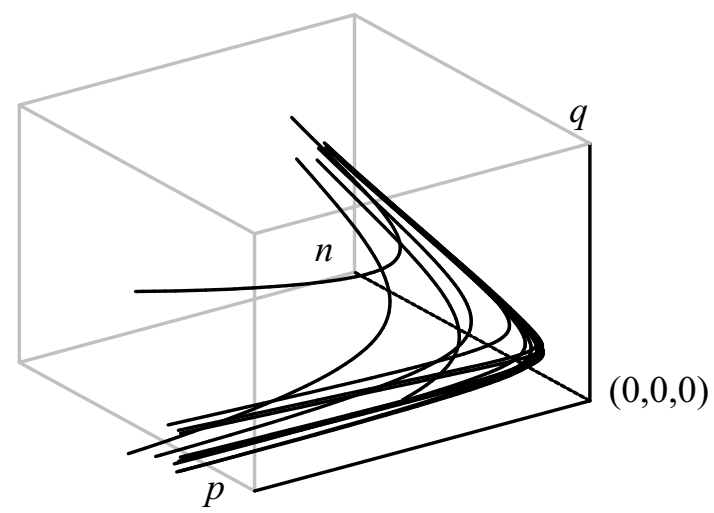

Figure 2 - Instability of $(0,0,0)$.

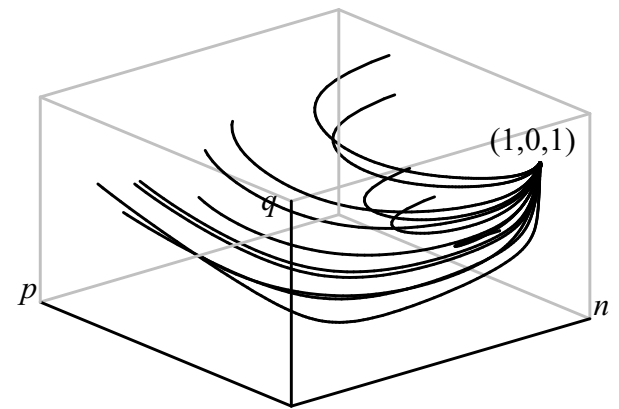

(a)

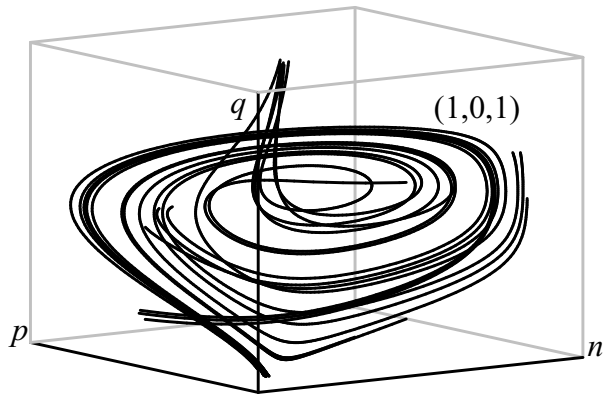

(b)

Figure 3 -Equilibrium $(1,0,1)$ : (a) Stable persistence. (b) Unstable persistence.

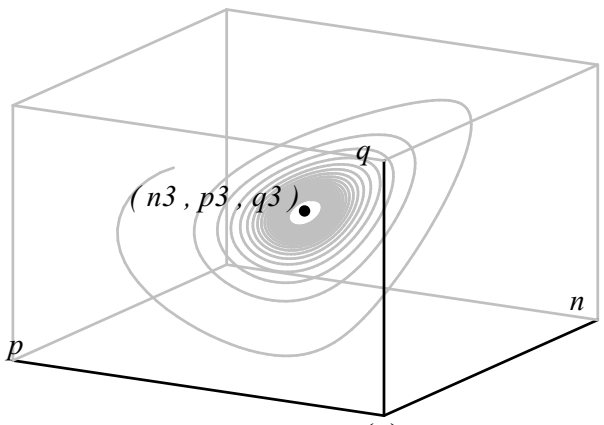

(a)

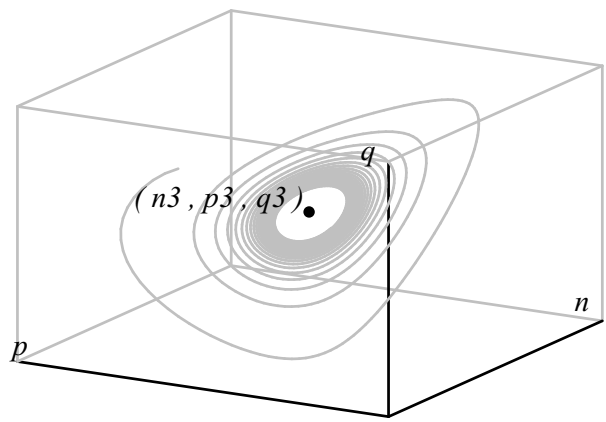

(b)

Figure $4-$ (a) Asymptotic stability at $a=0.985$. (b) Stable weak focus at $a_{0}=0.925$. 
respectively. Furthermore, decreasing the value of $a$ up to $a_{0}$ we see in Figure 4-(b) that the equilibrium $\left(n_{3}, p_{3}, q_{3}\right)$ is a weak stable focus.

The parameters given in the above paragraph we obtain Figures 5 (a) and (b) illustrating the occurrence of a periodic orbit around the equilibrium $\left(n_{3}, p_{3}, q_{3}\right)$ at $a=0.825$ and $a=0.414628$ respectively. Note that in this case we have $G_{4} \approx-2.595557406$ which shows numerically that the periodic orbit generated by the Hopf bifurcation is supercritical and $a_{0}=0.925$ is the bifurcation point. Finally, in Figure 6 we give a phase portrait for system (24), the restriction of system (4) to the center manifold.

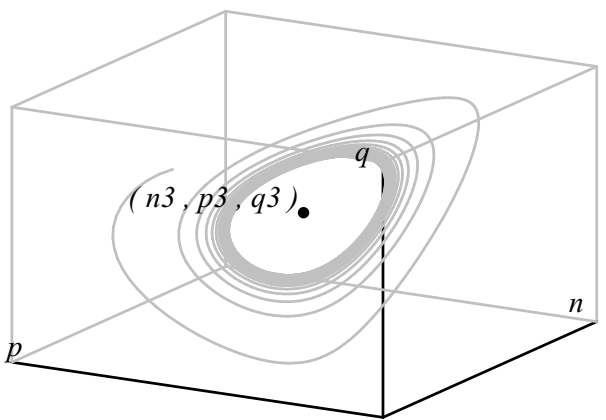

(a)

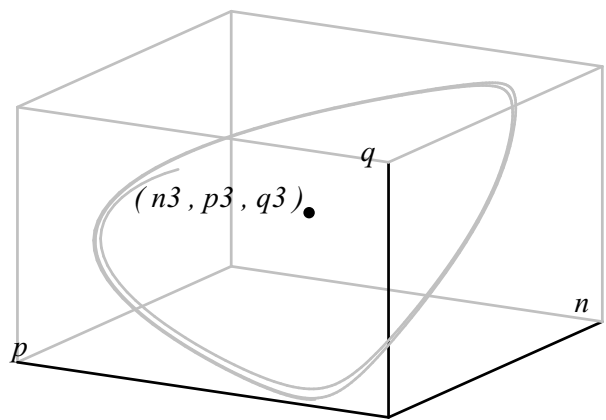

(b)

Figure 5 - (a) Periodic orbit at $a=0.825$. (b) Periodic orbit at $a=0.414628$.

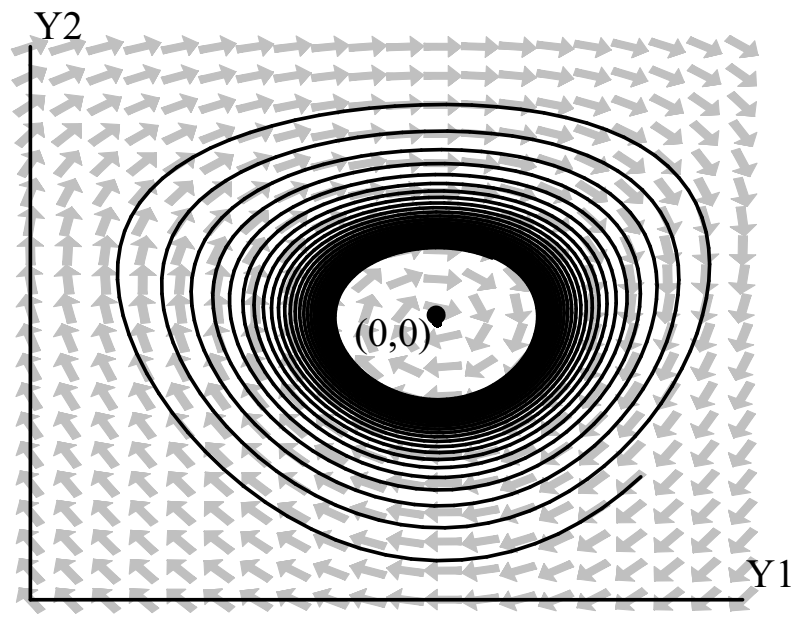

Figure 6 - System (4) restricted to the center manifold. 


\section{Maple Code}

In this section we include the main Maple code used to simulate Figure 2. The same code is appropriately modified for simulating other figures

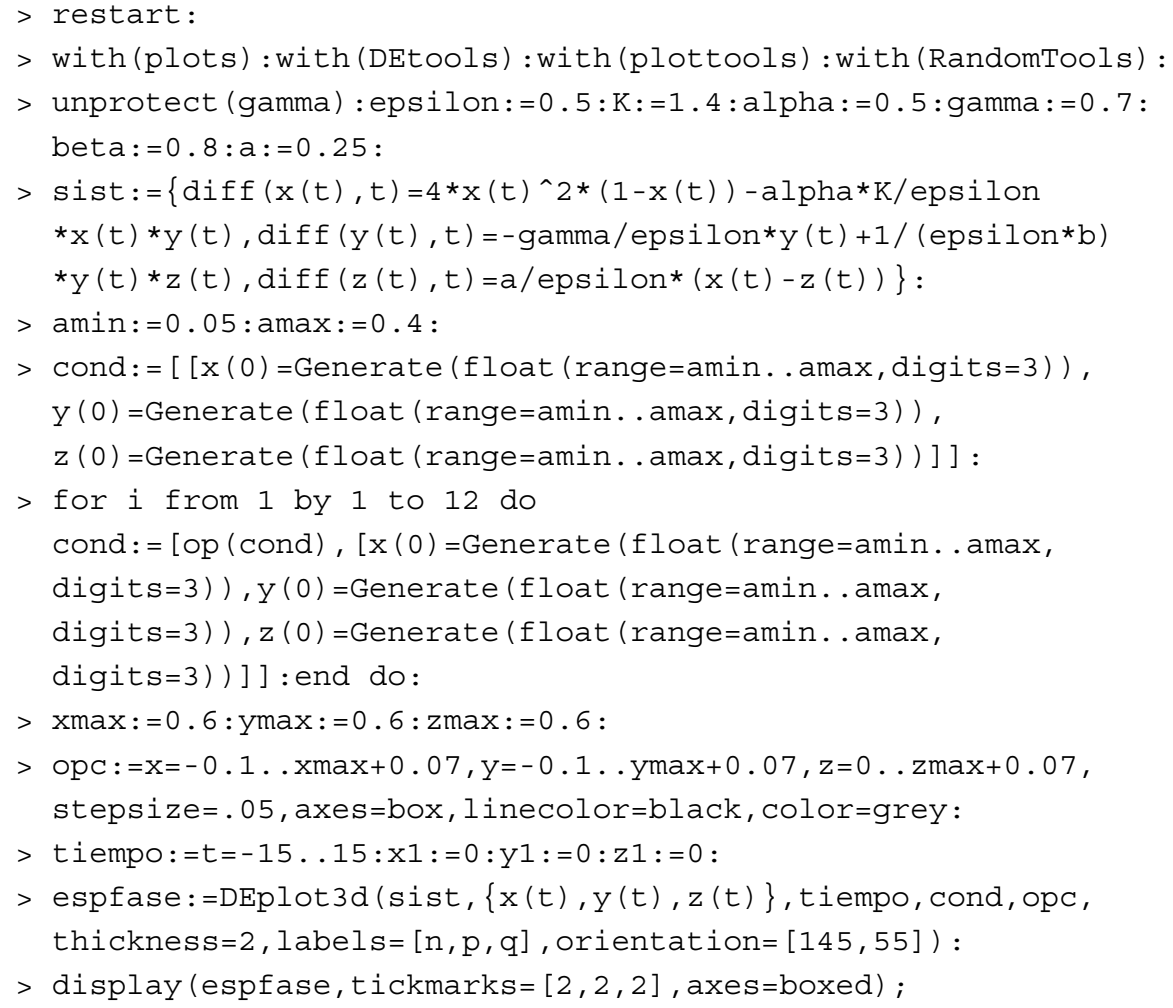

\section{Discussion}

In this paper we have studied a Lotka-Volterra predator-prey system introducing the weak Allee effect in the prey dynamics. A distributed delay is considered for the predator population. Aggregation and cooperation are common among various species in the nature. These characteristics are observed especially when the species search for food or when they try to escape from predators. The model studied is formulated along these lines. It is observed that this model exhibits interesting dynamics of the interacting populations due to the presence of the weak Allee effect different from the logistic growth models. 
Stability analysis of the equilibrium states is carried out. Conditions are derived for the occurrence of Hopf bifurcation of the equilibria of this model.

Farkas [8] has considered a predator-prey system similar to that of the present model with logistic type functional response and with weak Allee effect. The functional response considered in our model is different from that in [8]. Theorem 3.5 is an analogue of Theorem 7.3.1 (see [8]). It is observed that though the weak Allee effect drives the system to instability, the parameters associated with the memory will play a neutralizing role which is evident from the presence of periodic orbits.

Acknowledgments. The authors express their gratitude to late professor Miklós Farkas for the stimulating discussions. The authors wish to thank the anonymous reviewers for their constructive suggestions, which have led to the improvement of our earlier presentation.

\section{REFERENCES}

[1] A. Farkas, M. Farkas and L. Kaitar, On Hopf bifurcation in a predator-prey model. Diferential Equations: qualitative theory, pp. 283-290, Amsterdam, NorthHolland (1987).

[2] A. Farkas, M. Farkas and G. Szabo, Multiparameter bifurcation diagrams in predator-prey models with time lag. Journal of Mathematical Biology, 26 (1988), 93-106, Springer-Verlag.

[3] A. Bocsó and M. Farkas, Political and economic rationality leads to velcro bifurcation. Applied Mathematics and Computation, 140 (2003), 381-389.

[4] D. Boukal and L. Berec, Single-species models of the Allee effect: Extinction boundaries, sex ratios and mate enconunters. Journal of Theoretical Biology, 218 (2002), 375-394.

[5] J.M. Cushing, Two species competition in a periodic environment. Journal Mathematical Biology, 10 (1980), 385-400.

[6] K. Kiss and J. Tóth, n-dimensional ratio-dependent predator-prey systems with memory. Differential Equations and Dynamical Systems, 17(1-2) (2009), 11111141.

[7] L. Dai, Nonconstant periodic solutions in predator-prey systems with continuous time delays. Mathematical Bioscience, 53 (1981), 149-157. 
[8] M. Farkas, Periodic Motions. Springer-Verlag, New York (1994).

[9] M. Farkas, Dynamical Models in Biology. Academic Press, New York (2001).

[10] N. MacDonald, Time delay in a prey-predatormodels. II. Bifurcation theory. Mathematical Bioscience, 33 (1977), 227-234.

[11] H.I. Freedman and G.S.K. Wolkowicz, Predator-Prey Systems with Group Defence: The Paradox of Enrichment Revisited. Bulletin of Mathematical Biology, 48(516) (1986), 493-508. Printed in Great Britain.

[12] N. MacDonald, Time lags in biological models. Lecture Notes in Biomathematics 27, Springer-Verlag, Berlin (1978).

[13] S. Wiggins, Introduction to Applied Nonlinear Dynamical Systems and Chaos. Springer-Verlag, Heidelberg (1990).

[14] V. Volterra, Lecons sur la théorie mathématique de la lutte pour la vie. GauthierVillars, Paris (1931).

[15] W.C. Allee, Animal aggregations. Quart. Rev. Biol., 2 (1931), 367-398.

[16] W.C. Allee, Animal aggregations: A study in General Sociology. Chicago Univ. Press, Chicago (1993). 